\title{
Impact of cardiovascular disease on clinical outcomes in hospitalized patients with Covid-19: a systematic review and meta-analysis
}

\author{
Ernesto Maddaloni ${ }^{1}$ (1) - Luca D'Onofrio ${ }^{1} \cdot$ Antonio Siena $^{1} \cdot$ Cecilia Luordi $^{1} \cdot$ Carmen Mignogna ${ }^{1}$. \\ Rocco Amendolara ${ }^{1} \cdot$ Ilaria Cavallari $^{2} \cdot$ Francesco Grigioni $^{2} \cdot$ Raffaella Buzzetti $^{1}$
}

Received: 26 March 2021 / Accepted: 6 July 2021 / Published online: 17 July 2021

(c) The Author(s) 2021

\begin{abstract}
Contrasting data have been published about the impact of cardiovascular disease on Covid-19. A comprehensive synthesis and pooled analysis of the available evidence is needed to guide prioritization of prevention strategies. To clarify the association of cardiovascular disease with Covid-19 outcomes, we searched PubMed up to 26 October 2020, for studies reporting the prevalence of cardiovascular disease among inpatients with Covid-19 in relation to their outcomes. Pooled odds-ratios (OR) for death, for mechanical ventilation or admission in an intensive care unit (ICU) and for composite outcomes were calculated using random effect models overall and in the subgroup of people with comorbid diabetes. Thirty-three studies enrolling 52,857 inpatients were included. Cardiovascular disease was associated with a higher risk of death both overall (OR 2.58, $95 \%$ confidence intervals, CI 2.12-3.14, $p<0.001$, number of studies 24) and in the subgroup of people with diabetes (OR $2.91,95 \%$ CI 2.13-3.97, $p<0.001$, number of studies 4 ), but not with higher risk of ICU admission or mechanical ventilation (OR 1.35, 95\% CI 0.73-2.50, $p=0.34$, number of studies 4). Four out of five studies reporting OR adjusted for confounders failed to show independent association of cardiovascular disease with Covid-19 deaths. Accordingly, the adjusted-OR for Covid-19 death in people with cardiovascular disease dropped to 1.31 (95\% CI 1.01-1.70, $p=0.041$ ). Among patients hospitalized for Covid-19, cardiovascular disease confers higher risk of death, which was highly mitigated when adjusting the association for confounders.
\end{abstract}

Keywords Covid-19 $\cdot$ Pandemic $\cdot$ Cardiovascular disease $\cdot$ Meta-analysis

\section{Introduction}

Since its spread in late 2019, Coronavirus disease 2019 (Covid-19) caused more than 1 million deaths. Cardiometabolic risk factors, such as hypertension and diabetes are among the most frequent comorbidities in patients hospitalized for Covid-19. The mounting literature describing clinical features of patients with Covid-19 initially suggested that also pre-existing cardiovascular disease is an important risk factor for severe disease and death [1]. Nevertheless, our group and others failed to show significant associations between history of cardiovascular disease and poor Covid-19

Ernesto Maddaloni

ernesto.maddaloni@uniroma1.it

1 Department of Experimental Medicine, Sapienza University of Rome, Viale Regina Elena 324, 00161 Rome, Italy

2 Department of Cardiovascular Sciences, Campus Bio-Medico University of Rome, Rome, Italy outcomes, especially after adjustment for confounders [2-6]. Indeed, most of the available data are from small and underpowered studies differing in settings and features of the population enrolled. Therefore, a comprehensive synthesis and a pooled analysis of the rapidly increasing number of studies conducted in patients with Covid-19 are welcome to allow a better risk stratification and a more effective clinical care. In particular, it is important to disentangle whether and at what extent the presence of cardiovascular disease is associated with poor Covid-19 outcomes and if the impact of history of cardiovascular disease varies by countries and type of outcome. We also meant to understand if the existing evidence supports an association between cardiovascular disease and Covid-19 outcomes independently from confounders, such as older age and sex. To these aims, we conducted a systematic review and meta-analysis of studies reporting clinical outcomes of subjects hospitalized for Covid-19 with and without history of cardiovascular disease. We secondarily aimed to investigate whether cardiovascular disease further 
increases the risk of poor Covid-19 outcomes in the highrisk group of people with diabetes mellitus, which may be considered a cardiovascular equivalent.

\section{Methods}

\section{Search strategy and selection criteria}

In this systematic review and meta-analysis, we searched PubMed for the term "covid-19" looking for observational studies published in English language up to 26 October 2020 , reporting original clinical data about history of cardiovascular disease in Covid-19 inpatients aged $>18$ years old with and without at least one outcome among death, mechanical ventilation, admission in an intensive care unit (ICU), or a composite outcome with at least one of the above. The search was filtered to include only "clinical studies" and "observational studies". We excluded studies that were not original articles, randomized clinical trials testing the efficacy of therapeutical interventions on Covid-19 outcomes, whole population studies, studies conducted in nonhospitalized people, mathematical modeling and machine learning or computational studies.

Four investigators (AS, CM, CL and RA) independently screened titles, abstracts and full-text articles reporting potentially eligible studies. Disagreements were resolved by consultation with two adjudicators (EM and LDO) when necessary.

\section{Data collection}

Results in studies' reports and their accompanying supplementary materials were used as the only source of information. Databases of the individual studies were not obtained from the sponsoring institutions and analyses were performed at the study level. Data from each eligible article were independently extracted by one investigator (LDO, AS, CL, RA, CM) and entered in a structured spreadsheet. Data extraction was duplicated for all papers by two independent researchers (EM and IC). The following data were extracted: total number of participants, country of the hospital where patients were enrolled, definition of cardiovascular disease, outcomes of the study, number of patients with and without the study outcomes, number of patients with and without cardiovascular disease among patients with and without the study outcomes. Absolute numbers were recalculated when percentages were reported. Adjusted odds ratio (OR) with the corresponding 95\% confidence intervals (CI) were extracted if available.

\section{Outcomes}

The clinical outcomes evaluated in this meta-analysis were: death, mechanical ventilation or ICU admission, and a composite outcome with at least one of the above. If one study reported data for two outcomes among those above specified, data for both the outcomes were retrieved and analyzed. No study reported data for all the three above specified outcomes.

\section{Effect measures}

Crude OR and 95\% CI from each study were recalculated based on the absolute numbers of patients with and without cardiovascular disease among those with and without the study outcome. Adjusted OR and the corresponding 95\% CI were used instead of crude OR if available from the study.

\section{Data analysis}

The DerSimonian-Laird method for random effects [7] was used in the primary analyses to estimate the pooled OR for the three study outcomes, having history of cardiovascular disease (defined according to the definition reported in each study) as exposure. The DerSimonian-Laird method for random effects was also used to evaluate the pooled OR for death in the subgroup of subjects with diabetes mellitus. A separate meta-analysis including only studies reporting OR adjusted for confounders was also performed. $I^{2}$ was used to assess heterogeneity. Subgroup meta-analyses by country was conducted to explore heterogeneity. Countries represented in only one study (France, Greece, and Brazil) were grouped in the "other countries" subgroup. Publication bias was assessed visually using funnel plots and formally with Egger's test for the primary analyses if at least ten studies were included in the meta-analysis [8]. All meta-analyses were conducted using Stata version 12.1 (StataCorp, United States). $p$ values $<0.05$ were considered to be statistically significant.

\section{Results}

\section{Study selection}

We identified 638 articles in the published literature according to the search strategy used for this systematic review and meta-analysis (Fig. 1). We excluded 446 articles at the title/ abstract level because not in English, not on humans, reporting results of randomized clinical trials or study protocols. Of the remaining 192 articles assessed for eligibility at the 
Fig. 1 Flowchart of study selection

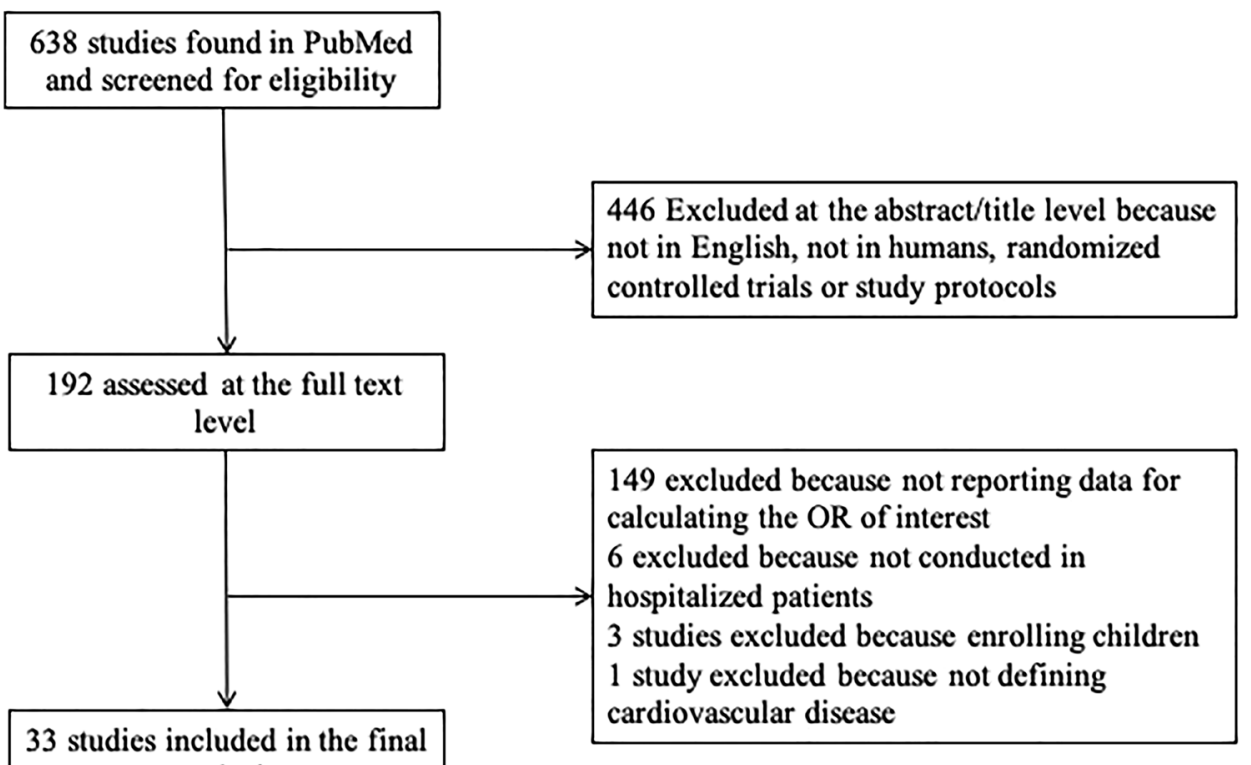

analysis full-text level, 149 did not report information useful for the calculation of the OR for any of the relevant outcomes in people with previous cardiovascular disease, 6 studies were not conducted in hospitalized patients, 3 studies enrolled children and 1 study did not define cardiovascular disease. Finally, 33 studies were included in this meta-analysis.

\section{Study characteristics}

The number of inpatients with available clinical data useful for this analysis in the 33 selected studies ranged from 52 to 20,133 , for a total of 52,857 hospitalized patients with Covid-19 included in this meta-analysis. Among these, $11,371(21.5 \%)$ had a positive history of cardiovascular disease. Twenty-three studies reported data useful for the calculation of the pooled OR for death [2, 4, 5, 9-28], four studies for the calculation of the pooled OR for ICU admission or mechanical ventilation $[6,29-31]$ and ten studies for the calculation of the pooled OR for any composite outcome including at least one among death, ICU admission or mechanical ventilation [2, 3, 5, 11, 21, 32-36] (Table 1). Since one study by Shi Q and colleagues [26] reported separate absolute numbers for people with and without diabetes mellitus, a total of 24 OR were considered for the calculation of the pooled OR for death.

The majority of studies were conducted in China $(n=11$, $33.3 \%)$, Italy $(n=6,18.2 \%)$ and United States $(n=5,15.2 \%)$. The remaining studies were from United Kingdom $(n=3)$, Korea $(n=2)$, Spain $(n=2)$, France $(n=1)$, Greece $(n=1)$, Poland $(n=1)$ and Brazil $(n=1)$. Cardiovascular disease was defined as "coronary heart disease" in 13 studies, while 15 studies did not better define the terms "cardiovascular disease" or "chronic heart disease". The remaining five studies defined "cardiovascular disease" as "major adverse cardiovascular events", as "ischemic or valvular heart disease", as a composite of "congestive heart failure, myocardial infarction or stroke", and as "acute myocardial infarction".

\section{Cardiovascular disease and Covid-19 outcomes}

Compared to Covid-19 hospitalized patients without, those with history of cardiovascular disease had a higher risk of death (pooled OR 2.56, 95\% CI 2.12-3.10, $p<0.001$, number of studies 24) but not of ICU admission or mechanical ventilation (pooled odds ratio $1.35,95 \%$ CI $0.73-2.50$, $p=0.34$, number of studies 4$)$; the pooled OR for composite outcomes was 1.72 (95\% CI 1.13-2.63, $p=0.011$, number of studies 10) (Fig. 2A-C). The heterogeneity was considerable among studies investigating death $\left(I^{2} 84.3 \%, p<0.001\right)$, while it was lower among studies investigating ICU admission or mechanical ventilation $\left(I^{2} 54.9 \%, p=0.084\right)$ and among those investigating composite outcomes ( $I^{2} 53.3 \%$, $p<0.001)$. No significant publication bias was found (Egger's tests: $p=0.18$ for death, $p=0.16$ for the composite outcome; funnel plots in supplementary figures S1, S2; publication bias was not formally tested for the outcome ICU admission or mechanical ventilation because less than ten studies were included).

Five studies reported OR for poor Covid-19 outcomes adjusted for confounders. Among these, only Kim DW et al. reported a significant 2.38 -fold increased risk of death $(95 \%$ CI 1.03-5.49) among patients with previous acute myocardial infarction after adjusting for sex, age, type of districts, high epidemic region and socio-economic status [14]. On the 


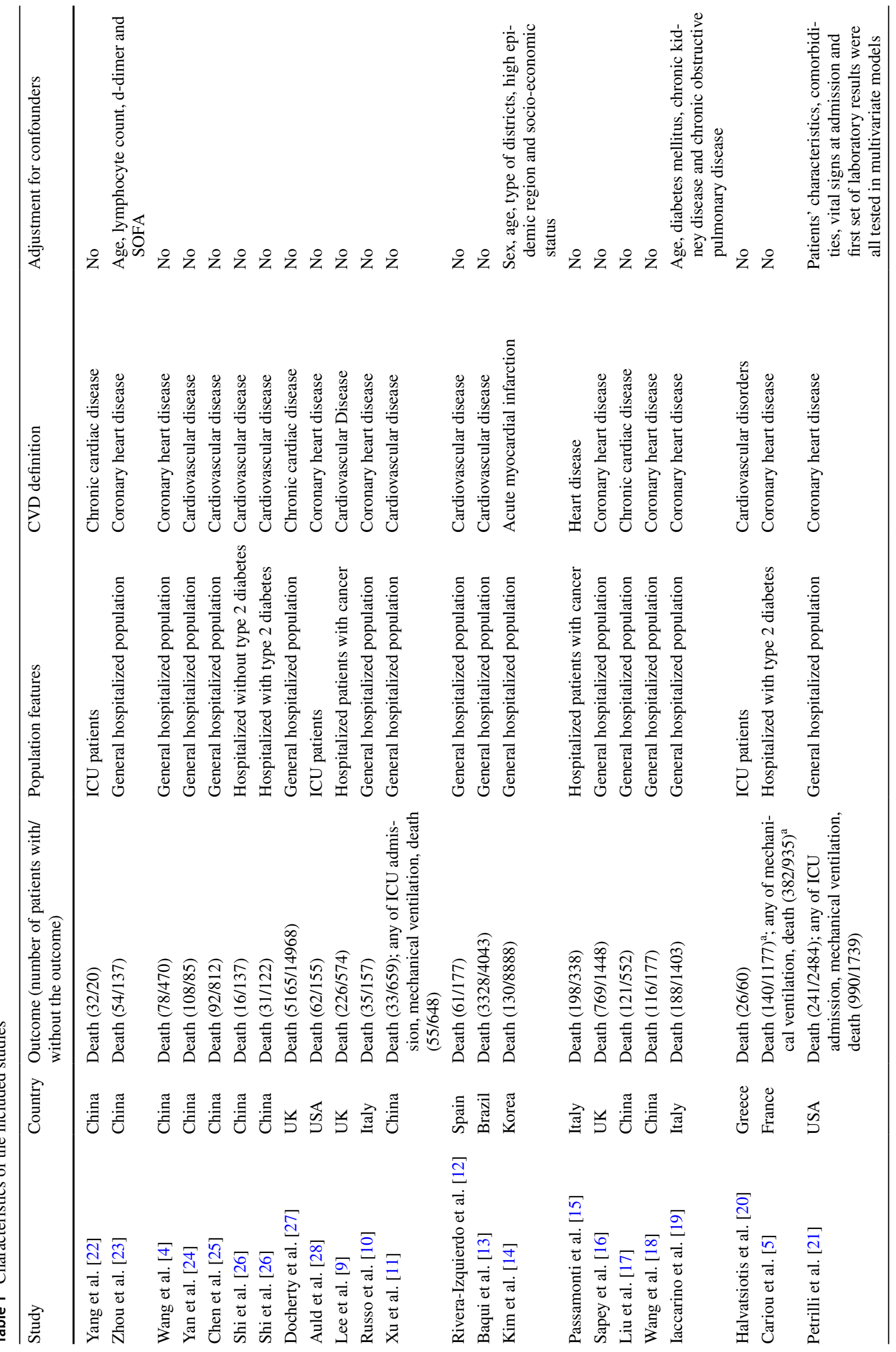




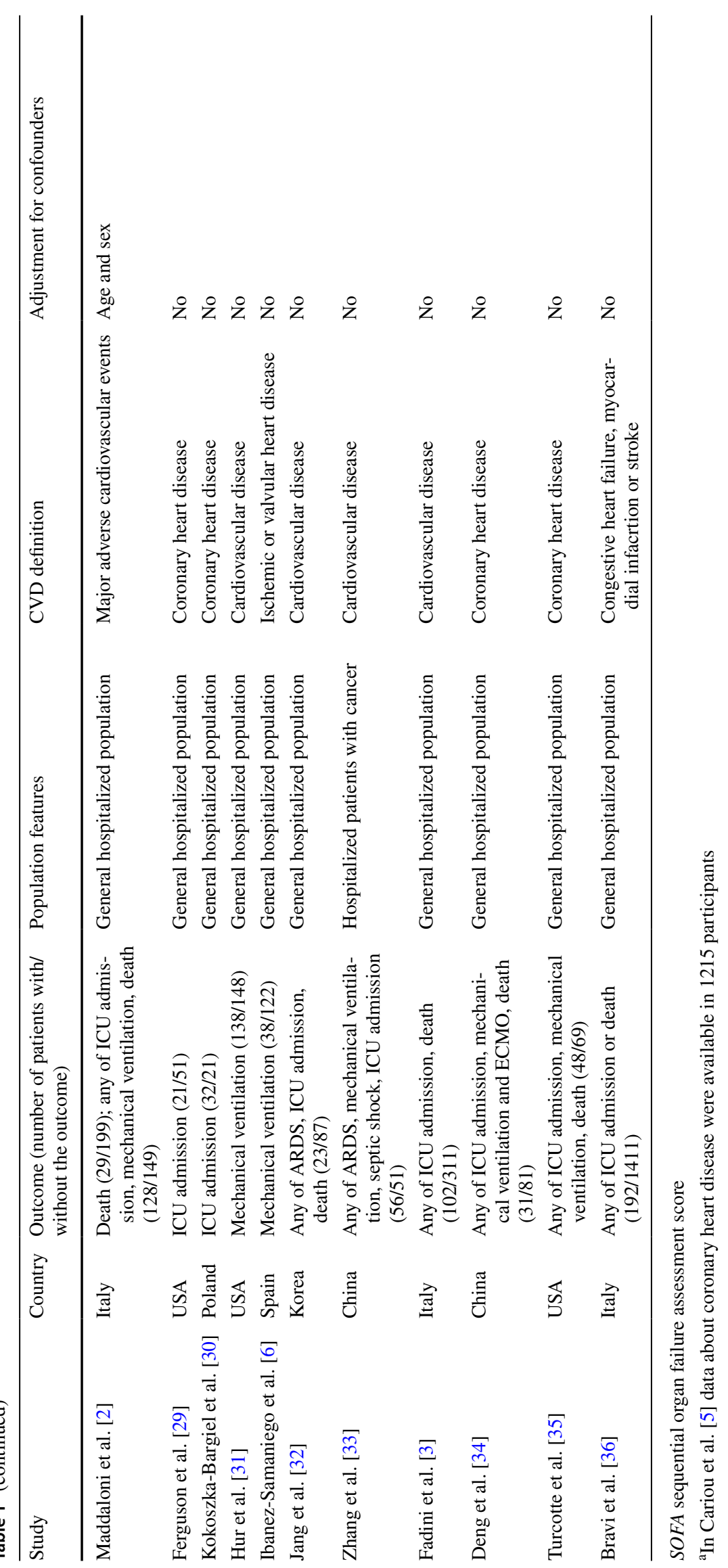




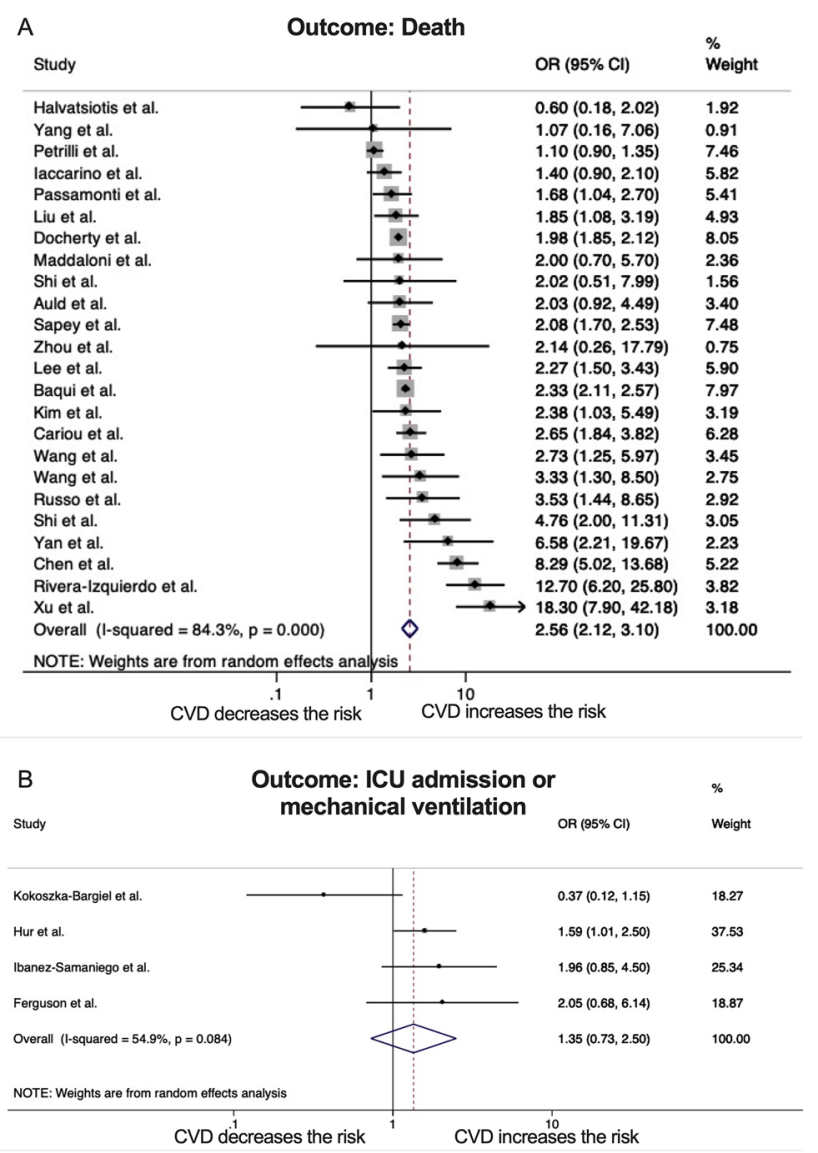

C

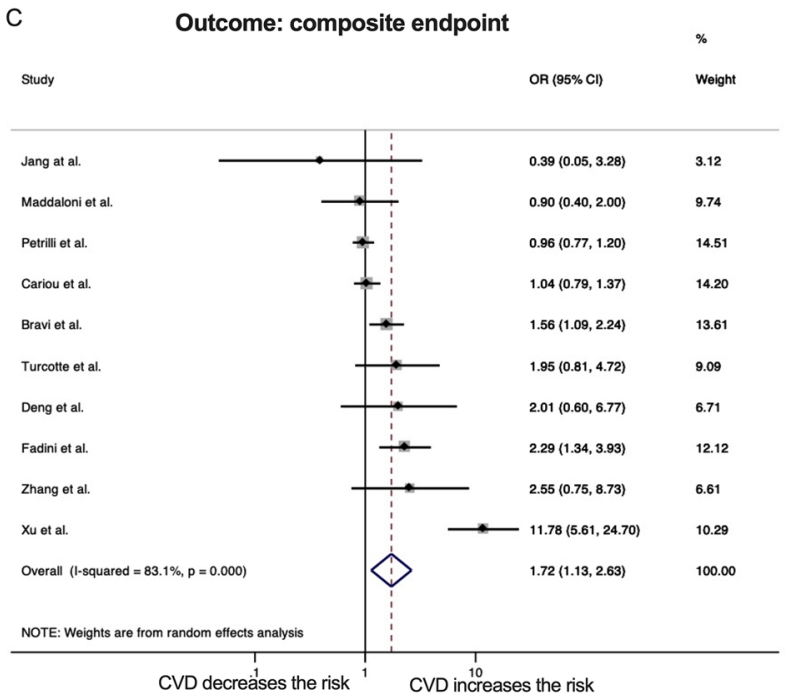

Fig. 2 Forrest plots of pooled risks of death (A), mechanical ventilation or ICU admission (B) and composite outcomes including at least one of the above (C) among inpatients with Covid-19 and positive history of cardiovascular disease compared to those without cardiovascular disease. Abbreviations: OR, odds ratio; $\mathrm{CI}$, confidence intervals; CVD, cardiovascular disease contrary, Zhou et al. [23] and Iaccarino et al. [19] did not find independent associations with death. Similarly, cardiovascular disease was not independently associated both with the respective composite primary outcomes and with death in Petrilli et al. [21] and in Maddaloni et al. [2]. Accordingly, the meta-analysis showed that cardiovascular disease was not independently associated with the primary outcomes of these studies (pooled OR 1.20, 95\% CI 0.87-1.66, $p=0.26$ ), and the pooled adjusted-OR for death among inpatients with cardiovascular disease decreased to 1.31 (95\% CI 1.01-1.70, $p=0.041, I^{2} 19.6 \%, p=0.29$ ) (Fig. 3).

\section{Meta-analyses by country}

To explore the heterogeneity found among studies evaluating the risk of death, pooled ORs by country were calculated and confirmed an increased risk of death among inpatients with cardiovascular disease hospitalized in all countries, but in Greece (OR 0.69, 95\% CI 0.18-2.02) [20] and in USA (pooled OR 1.32, 95\% CI 0.76-2.28). Of note, the analysis by countries seemed to explain at least in part the heterogeneity found in the primary meta-analysis for death, remaining considerable only for studies from China $\left(I^{2} 72.9 \%\right.$, $p<0.001$ ) (Fig. 4).

\section{Cardiovascular disease and Covid-19 in patients with type 2 diabetes}

Four studies reported data about the prevalence of cardiovascular disease among Covid-19 survivors and non-survivors with comorbid type 2 diabetes [5, 24-26]. Overall, the presence of cardiovascular disease on top of diabetes was associated with a 2.9-fold higher risk of death (pooled OR 2.91, 95\% CI 2.13-3.97, $p<0.001$ ) (Fig. 5). Cariou et al. [5] also reported data about the risk of a composite outcome of mechanical ventilation or death within 7 days of admission among people with comorbid diabetes and found no significant association of cardiovascular disease with the composite outcome.

\section{Discussion}

This systematic review and meta-analysis of observational studies conducted among hospitalized patients with Covid-19 shows that those with history of cardiovascular disease are, on average, 2.58-times more likely to die than those without, while no significant increase in the risk of mechanical ventilation or ICU admission was found. When restricting the analysis to include studies adjusting results for 


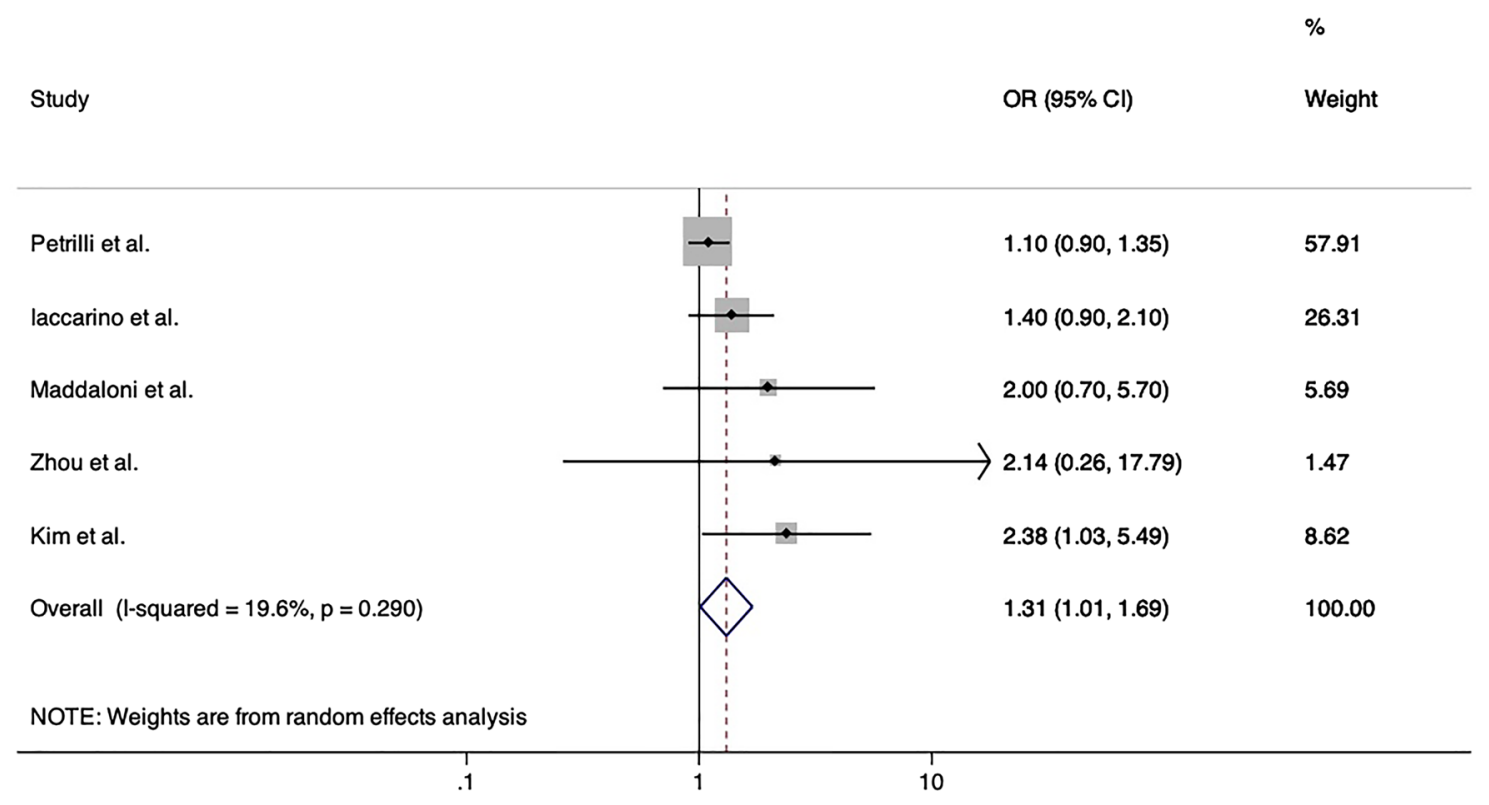

Fig. 3 Forrest plot of pooled adjusted risk of death among inpatients with Covid-19 and positive history of cardiovascular disease compared to those without. Abbreviations: OR, odds ratio; CVD, cardiovascular disease

confounders such as age, sex and other comorbidities, the impact of cardiovascular disease on poor Covid-19 outcomes was reduced. Overall, our results suggest that cardiovascular disease is the tip of the iceberg of several cardiovascular factors contributing to the severity of Covid-19.

Accordingly, crucial mechanisms that have been hypothesized to explain the high rates of Covid-19 progression towards critical scenarios, or even death, may be enhanced by cardiometabolic conditions. In particular, the pro-thrombotic and pro-inflammatory milieu predisposing cardiometabolic patients to cardiovascular events [37] may also promote the cytokine storm and the formation of multiple blood clots that can occur in the most severe Covid-19 cases [38, 39]. Indeed, thrombotic complications are frequent and significantly contribute to morbidity and mortality among Covid-19 patients [40, 41]. In this regard, differences in thromboprophylaxis, which has been indicated in ICU patients, in those with acute respiratory insufficiency and in the presence of mildto-moderate respiratory symptoms and an elevated risk of venous thromboembolism [38, 42], may exist. However, most of the studies published so far did not adjust their observations for confounders, potentially leading to deceiving conclusions. Therefore, we also investigated this association gathering data only from studies conducting multivariate analyses which allow to understand the relevance of considering such confounders when evaluating the role of cardiovascular disease in Covid-19 progression. Of note, we found results corrected for confounders in only 5 studies out of the 33 (15.2\%) included, and almost all (4 out of 5) failed to show independent associations of cardiovascular disease with Covid-19 deaths or composite outcomes. Accordingly, the adjusted pooled OR for death was more than 1 point lower compared to the crude pooled OR. However, the heterogeneity of adjustments between studies should be acknowledged as a limitation of this meta-analysis.

Another finding of this meta-analysis is the heterogeneity of the prognostic impact of cardiovascular disease on Covid19 observed among different countries. Possible explanations to this result may rely in different secondary prevention strategies in various healthcare systems, in different criteria used for hospitalizing people affected by Covid-19 or in a role for ethnicity.

Differently from what observed for death, no association between cardiovascular disease and risk of ICU admission or mechanical ventilation was found. This observation may lead to the hypothesis that cardiovascular disease impacts on disease progression among patients affected by the most severe cases of Covid-19, who are at the highest risk of death, but not among people affected by moderate or mild Covid-19. However, we were not able to perform a sensitivity analysis by subgroups of Covid- 19 severity because of the lack of such information in the available literature.

Finally, we evaluated whether cardiovascular disease increases the risk of poor Covid-19 outcomes in subjects with type 2 diabetes confirming the association found in the general population when using crude OR. This result is not consistent with a previous study conducted by our group reporting that the presence of cardiovascular disease was not 


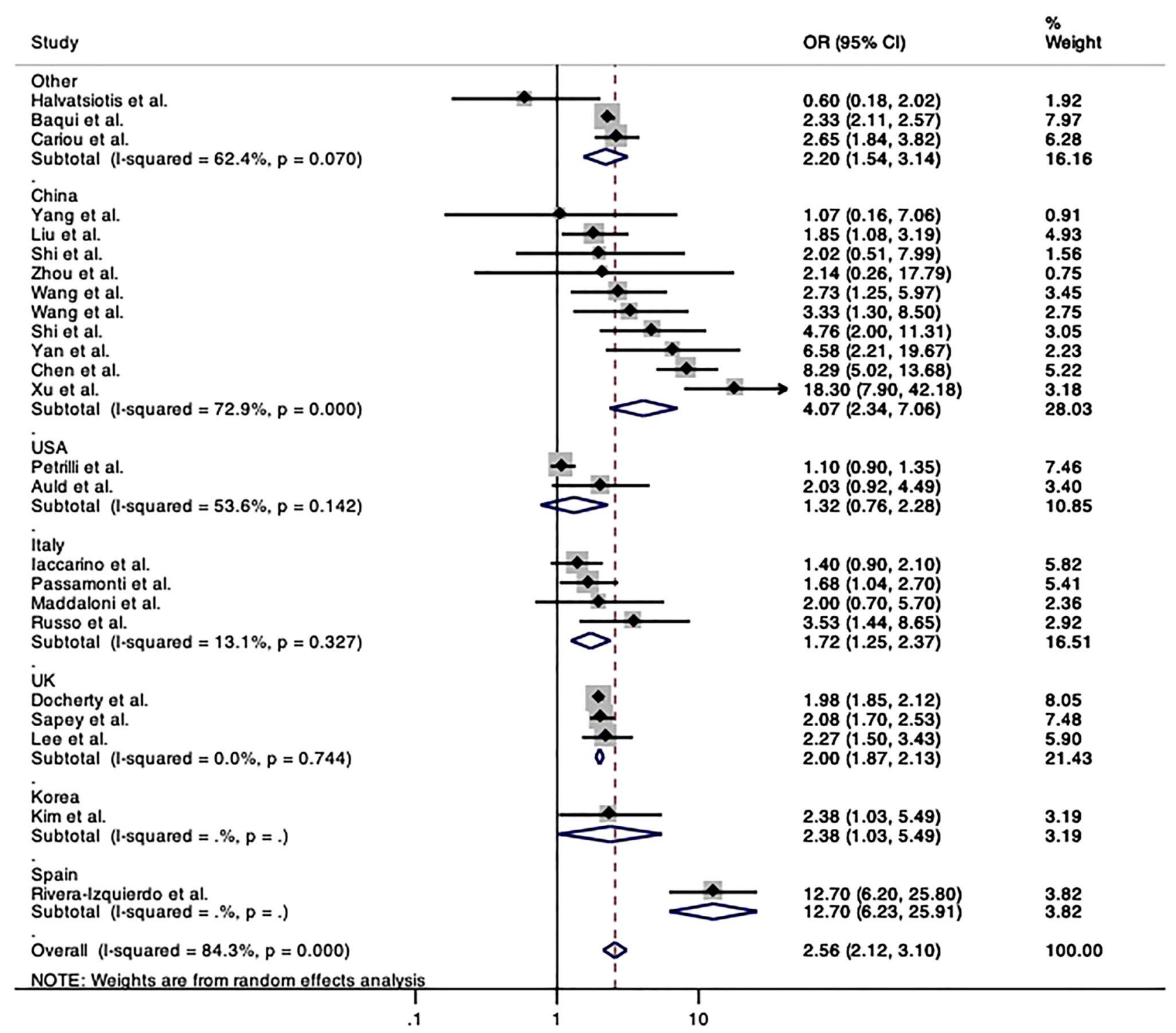

Fig. 4 Forrest plot of pooled risk of death among inpatients with Covid-19 and positive history of cardiovascular disease compared to those without by country. Abbreviations: OR, odds ratio; CI, confidence intervals; CVD, cardiovascular disease

associated to Covid-19 hospitalization among people with type 2 diabetes [43]. However, the different outcome and the fact that correction for confounders was not performed in any study reporting data in the subgroup of people with diabetes may explain this apparent contrast.

Strengths of this study include the systematic review of published papers with available data helping to disentangle the complex association between cardiovascular disease and Covid-19 outcomes [44], the gathering of data from a high number studies from different countries including more than 50,000 inpatients and the identification and separate analysis of studies reporting adjusted associations to better clarify the real impact of cardiovascular disease on Covid-19 outcomes. Nevertheless, some limitations should be acknowledged.
Our search was limited to studies published in PubMed and, therefore, we might have missed papers published in EMBASE, Cochrane Library, PROSPERO or other databases. Differences across papers with regards to populations and explored outcomes and the often-vague definition of cardiovascular disease resulted in high heterogeneity. However, this does not preclude pooling of data, it is consistent with other meta-analyses on Covid-19 [45], and heterogeneity was explored through subgroup analyses. Instead, our study provides a reliable outlook of the available data, highlights the heterogeneity across the Covid-19 literature and the need to improve the quality and standardization of research in this field. Specifically, a clearer definition of cardiovascular disease is needed when reporting data about the risk factors 


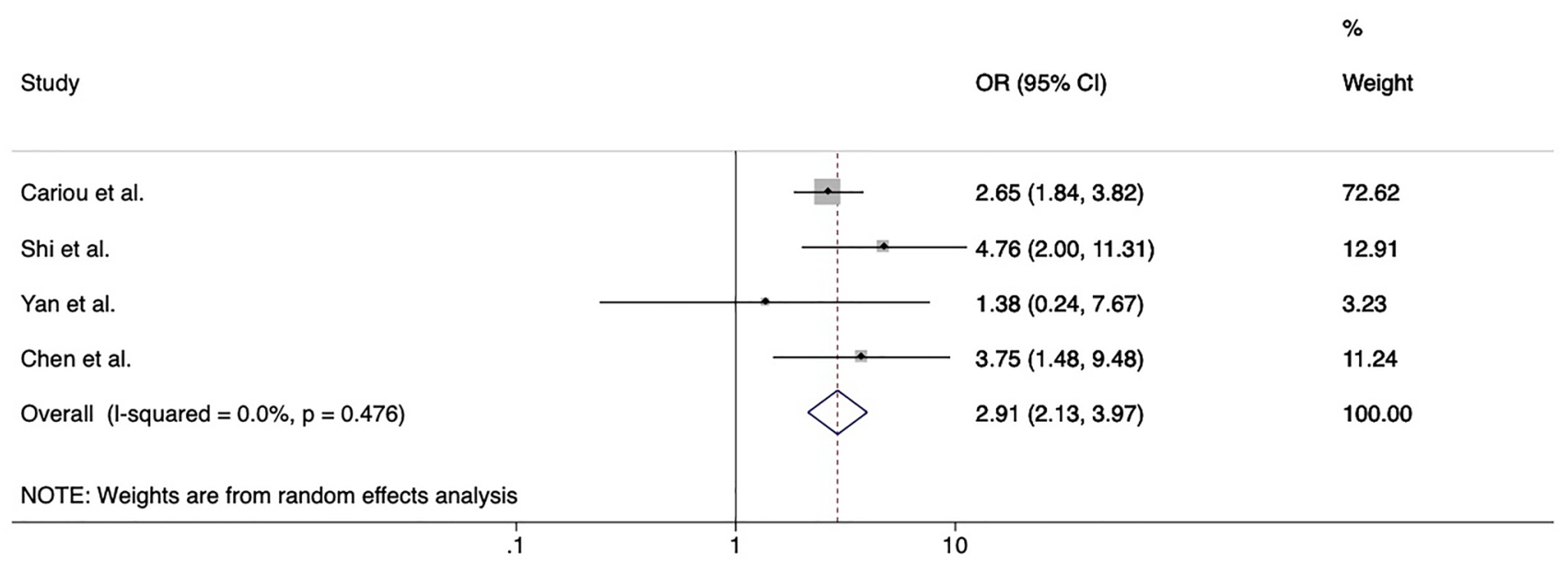

Fig. 5 Forrest plot of pooled risk of death among inpatients with diabetes mellitus comparing patients with and without positive history of cardiovascular disease. Abbreviations: OR, odds ratio; CI, confidence intervals; CVD, cardiovascular disease

for poor Covid-19 prognosis. Indeed, our systematic review and meta-analysis shows that about a half of the included studies does not clearly define "history of cardiovascular disease", possibly including a highly heterogeneous population within the group of people with the disease. In this regard, this study did not specifically investigate the impact of heart failure on Covid-19 outcomes, which should deserve a separate meta-analysis. It is also important to highlight the high heterogeneity we found in the literature with regards to the definitions of poor Covid-19 outcomes, claiming for a widely agreed consensus to standardize the analysis of clinical data around the globe. Finally, despite this systematic review and meta-analysis being conducted in the late phases of the Covid-19 pandemic, we believe that these results are still of value to guide prioritization of certain patients for primary and secondary Covid-19 prevention. Unfortunately, time is still needed before the pandemic will be definitely defeated and future infectious diseases by pathogens similar to SARS-CoV-2 could spread.

\section{Conclusions}

Among patients hospitalized for Covid-19, cardiovascular disease confers higher risk of death, which is mostly explained when adjusting for confounders, but not of mechanical ventilation or ICU admission. Since the majority of the studies with multivariate analyses failed to show an independent role of cardiovascular disease to increase the risk of Covid-19 progression towards poor outcomes, potential explanations for the higher prevalence of cardiovascular disease among patients suffering from severe Covid-19 should be mostly searched in cardiovascular risk factors rather than cardiovascular disease itself. These may include ageing, the increased frailty of patients with comorbid cardiovascular disease or, most probably, the comorbidities often co-existing with and predisposing to cardiovascular events, such as obesity, diabetes and hypertension.

Supplementary Information The online version contains supplementary material available at https://doi.org/10.1007/s11739-021-02804-x.

Funding Open access funding provided by Università degli Studi di Roma La Sapienza within the CRUI-CARE Agreement. No funding source to declare.

Availability of data and material All data used in this manuscript can be found in the online versions of the studies that were accessed. Our own data synthesis of these manuscripts is available from the corresponding author upon reasonable request.

\section{Declarations}

Conflict of interest The authors declare no conflict of interest related to this manuscript.

Human and animal rights and informed consent This is an analysis of previous research data and uses data that existed in studies published. Therefore neither ethics approval nor patient consent was required for this analysis.

Open Access This article is licensed under a Creative Commons Attribution 4.0 International License, which permits use, sharing, adaptation, distribution and reproduction in any medium or format, as long as you give appropriate credit to the original author(s) and the source, provide a link to the Creative Commons licence, and indicate if changes were made. The images or other third party material in this article are included in the article's Creative Commons licence, unless indicated otherwise in a credit line to the material. If material is not included in the article's Creative Commons licence and your intended use is not permitted by statutory regulation or exceeds the permitted use, you will need to obtain permission directly from the copyright holder. To view a copy of this licence, visit http://creativecommons.org/licenses/by/4.0/. 


\section{References}

1. Clerkin KJ, Fried JA, Raikhelkar J et al (2020) COVID-19 and cardiovascular disease. Circulation 141:1648-1655. https://doi. org/10.1161/CIRCULATIONAHA.120.046941

2. Maddaloni E, D'Onofrio L, Alessandri F et al (2020) Cardiometabolic multimorbidity is associated with a worse Covid-19 prognosis than individual cardiometabolic risk factors: a multicentre retrospective study (CoViDiab II). Cardiovasc Diabetol. https:// doi.org/10.1186/s12933-020-01140-2

3. Fadini GP, Morieri ML, Boscari F et al (2020) Newly-diagnosed diabetes and admission hyperglycemia predict COVID-19 severity by aggravating respiratory deterioration. Diabetes Res Clin Pract 168:108374. https://doi.org/10.1016/j.diabres.2020.108374

4. Wang K, Zhang Z, Yu M et al (2020) 15-day mortality and associated risk factors for hospitalized patients with COVID-19 in Wuhan, China: an ambispective observational cohort study. Intensive Care Med 46:1472-1474. https://doi.org/10.1007/ s00134-020-06047-w

5. Cariou B, Hadjadj S, Wargny M et al (2020) Phenotypic characteristics and prognosis of inpatients with COVID-19 and diabetes: the CORONADO study. Diabetologia. https://doi.org/10.1007/ s00125-020-05180-x

6. Ibáñez-Samaniego L, Bighelli F, Usón C et al (2020) Elevation of liver fibrosis index FIB-4 is associated with poor clinical outcomes in patients with COVID-19. J Infect Dis 222:726-733. https://doi.org/10.1093/infdis/jiaa355

7. DerSimonian R, Laird N (1986) Meta-analysis in clinical trials. Control Clin Trials 7:177-188. https://doi.org/10.1016/01972456(86)90046-2

8. Egger M, Smith GD, Schneider M, Minder C (1997) Bias in metaanalysis detected by a simple, graphical test. BMJ 315:629-634. https://doi.org/10.1136/bmj.315.7109.629

9. Lee LYW, Cazier JB, Angelis V et al (2020) COVID-19 mortality in patients with cancer on chemotherapy or other anticancer treatments: a prospective cohort study. Lancet. https://doi.org/10. 1016/S0140-6736(20)31173-9

10. Russo V, Di Maio M, Attena E et al (2020) Clinical impact of pre-admission antithrombotic therapy in hospitalized patients with COVID-19: a multicenter observational study. Pharmacol Res. https://doi.org/10.1016/j.phrs.2020.104965

11. Xu PP, Tian RH, Luo S et al (2020) Risk factors for adverse clinical outcomes with COVID-19 in China: a multicenter, retrospective, observational study. Theranostics. https://doi.org/10.7150/ thno. 46833

12. Rivera-Izquierdo M, del Carmen Valero Ubierna M, DelAmo JLR et al (2020) Sociodemographic, clinical and laboratory factors on admission associated with COVID-19 mortality in hospitalized patients: A retrospective observational study. PLoS ONE. https:// doi.org/10.1371/journal.pone.0235107

13. Baqui P, Bica I, Marra V et al (2020) Ethnic and regional variations in hospital mortality from COVID-19 in Brazil: a crosssectional observational study. Lancet Glob Health. https://doi.org/ 10.1016/S2214-109X(20)30285-0

14. Kim DW, Byeon KH, Kim J et al (2020) The correlation of comorbidities on the mortality in patients with COVID-19: an observational study based on the Korean National Health Insurance Big Data. J Korean Med Sci. https://doi.org/10.3346/jkms.2020.35. e243

15. Passamonti F, Cattaneo C, Arcaini L et al (2020) Clinical characteristics and risk factors associated with COVID-19 severity in patients with haematological malignancies in Italy: a retrospective, multicentre, cohort study. Lancet Haematol. https://doi.org/ 10.1016/S2352-3026(20)30251-9
16. Sapey E, Gallier S, Mainey C et al (2020) Ethnicity and risk of death in patients hospitalised for COVID-19 infection in the UK: an observational cohort study in an urban catchment area. BMJ Open Respir Res. https://doi.org/10.1136/bmjresp-2020-000644

17. Liu FY, Sun XL, Zhang Y et al (2020) Evaluation of the risk prediction tools for patients with coronavirus disease 2019 in Wuhan, China: a single-centered, retrospective, observational study. Crit Care Med. https://doi.org/10.1097/CCM.0000000000004549

18. Wang Z, Ye D, Wang M et al (2020) Clinical features of COVID19 patients with different outcomes in Wuhan: a retrospective observational study. Biomed Res Int. https://doi.org/10.1155/ 2020/2138387

19. Iaccarino G, Grassi G, Borghi C et al (2020) Age and multimorbidity predict death among COVID-19 patients. Hypertension 76:366-372. https://doi.org/10.1161/HYPERTENSIONAHA. 120.15324

20. Halvatsiotis P, Kotanidou A, Tzannis K et al (2020) Demographic and clinical features of critically ill patients with COVID-19 in Greece: the burden of diabetes and obesity. Diabetes Res Clin Pract. https://doi.org/10.1016/j.diabres.2020.108331

21. Petrilli CM, Jones SA, Yang J et al (2020) Factors associated with hospital admission and critical illness among 5279 people with coronavirus disease 2019 in New York City: prospective cohort study. BMJ. https://doi.org/10.1136/bmj.m1966

22. Yang X, Yu Y, Xu J et al (2020) Clinical course and outcomes of critically ill patients with SARS-CoV-2 pneumonia in Wuhan, China: a single-centered, retrospective, observational study. Lancet Respir Med. https://doi.org/10.1016/S2213-2600(20)30079-5

23. Zhou F, Yu T, Du R et al (2020) Clinical course and risk factors for mortality of adult inpatients with COVID-19 in Wuhan, China: a retrospective cohort study. Lancet 395:1054-1062. https://doi. org/10.1016/S0140-6736(20)30566-3

24. Yan Y, Yang Y, Wang F et al (2020) Clinical characteristics and outcomes of patients with severe covid-19 with diabetes. BMJ Open Diabetes Res Care 8:e001343. https://doi.org/10.1136/ bmjdrc-2020-001343

25. Chen Y, Yang D, Cheng B (2020) Clinical characteristics and outcomes of patients with diabetes and COVID-19 in association with glucose-lowering medication. Diabetes Care. https://doi.org/ $10.2337 / \mathrm{dc} 20-0660$

26. Shi Q, Zhang XX, Jiang F et al (2020) Clinical characteristics and risk factors for mortality of COVID-19 patients with diabetes in Wuhan, China: a two-center, retrospective study. Diabetes Care 43:1382-1391. https://doi.org/10.2337/dc20-0598

27. Docherty AB, Harrison EM, Green CA et al (2020) Features of 20133 UK patients in hospital with covid-19 using the ISARIC WHO clinical characterisation protocol: prospective observational cohort study. BMJ. https://doi.org/10.1136/bmj.m1985

28. Auld SC, Caridi-Scheible M, Blum JM et al (2020) ICU and ventilator mortality among critically Ill adults with coronavirus disease 2019*. Crit Care Med. https://doi.org/10.1097/CCM.0000000000 004457

29. Ferguson J, Rosser JI, Quintero O et al (2020) Characteristics and outcomes of coronavirus disease patients under nonsurge conditions, Northern California, USA, March-April 2020. Emerg Infect Dis. https://doi.org/10.3201/eid2608.201776

30. Kokoszka-Bargieł I, Cyprys P, Rutkowska K et al (2020) Intensive care unit admissions during the first 3 months of the COVID-19 pandemic in Poland: a single-center, cross-sectional study. Med Sci Monit. https://doi.org/10.12659/MSM.926974

31. Hur K, Price CPE, Gray EL et al (2020) Factors associated with intubation and prolonged intubation in hospitalized patients with COVID-19. Otolaryngol Head Neck Surg (United States). https:// doi.org/10.1177/0194599820929640 
32. Jang JG, Hur J, Choi EY et al (2020) Prognostic factors for severe coronavirus disease 2019 in Daegu, Korea. J Korean Med Sci. https://doi.org/10.3346/JKMS.2020.35.E209

33. Zhang H, Wang L, Chen Y et al (2020) Outcomes of novel coronavirus disease 2019 (COVID-19) infection in 107 patients with cancer from Wuhan, China. Cancer. https://doi.org/10.1002/cncr. 33042

34. Deng Q, Hu B, Zhang Y et al (2020) Suspected myocardial injury in patients with COVID-19: evidence from front-line clinical observation in Wuhan, China. Int J Cardiol. https://doi.org/10. 1016/j.ijcard.2020.03.087

35. Turcotte JJ, Meisenberg BR, MacDonald JH et al (2020) Risk factors for severe illness in hospitalized Covid-19 patients at a regional hospital. PLoS ONE. https://doi.org/10.1371/journal. pone. 0237558

36. Bravi F, Flacco ME, Carradori T et al (2020) Predictors of severe or lethal COVID-19, including angiotensin converting enzyme inhibitors and angiotensin ii receptor blockers, in a sample of infected Italian citizens. PLoS ONE 15:e0235248. https://doi.org/ 10.1371/journal.pone.0235248

37. Patti G, Cavallari I, Andreotti F et al (2018) Prevention of atherothrombotic events in patients with diabetes mellitus: from antithrombotic therapies to new-generation glucoselowering drugs. Nat Rev Cardiol. https://doi.org/10.1038/ s41569-018-0080-2

38. Patti G, Lio V, Cavallari I et al (2020) Questions and answers on practical thrombotic issues in SARS-CoV-2 infection: a guidance document from the Italian Working Group on atherosclerosis, thrombosis and vascular biology. Am J Cardiovasc Drugs 20:559-570. https://doi.org/10.1007/s40256-020-00446-6

39. Leisman DE, Deutschman CS, Legrand M (2020) Facing COVID19 in the ICU: vascular dysfunction, thrombosis, and dysregulated inflammation. Intensive Care Med. https://doi.org/10.1007/ s00134-020-06059-6

40. Malas MB, Naazie IN, Elsayed N et al (2020) Thromboembolism risk of COVID-19 is high and associated with a higher risk of mortality: a systematic review and meta-analysis. EClinicalMedicine 29-30:100639. https://doi.org/10.1016/j.eclinm.2020.100639

41. Scudiero O, Lombardo B, Brancaccio M et al (2021) Exercise, immune system, nutrition, respiratory and cardiovascular diseases during COVID-19: a complex combination. Int J Environ Res Public Health 18:904. https://doi.org/10.3390/ijerph18030904

42. Patti G, Lio V, Cavallari I et al (2020) Terapie antitrombotiche in pazienti con infezione da SARS-CoV-2: dalle attuali evidenze alle ragionevoli raccomandazioni-Position paper del Gruppo di Studio Aterosclerosi, Trombosi e Biologia Vascolare. G Ital Cardiol 21:489-501. https://doi.org/10.1714/3386.33634

43. Maddaloni E, D'Onofrio L, Alessandri F et al (2020) Clinical features of patients with type 2 diabetes with and without Covid19: a case control study (CoViDiab I). Diabetes Res Clin Pract. https://doi.org/10.1016/j.diabres.2020.108454

44. Juutilainen A, Lehto S, Ronnemaa T et al (2005) Type 2 Diabetes as a "Coronary Heart Disease Equivalent": an 18-year prospective population-based study in Finnish subjects. Diabetes Care 28:2901-2907. https://doi.org/10.2337/diacare.28.12.2901

45. Sze S, Pan D, Nevill CR et al (2020) Ethnicity and clinical outcomes in COVID-19: a systematic review and meta-analysis. EClinicalMedicine 29-30:100630. https://doi.org/10.1016/j. eclinm.2020.100630

Publisher's Note Springer Nature remains neutral with regard to jurisdictional claims in published maps and institutional affiliations. 